\title{
Comportamento Dinâmico de Estruturas Reticuladas com Juntas Semi-rígidas
}

\section{Dynamic Behaviour of Plane Frames with Semi-rigid Connections}

\author{
${ }^{1}$ Milton Soares Filho \\ ${ }^{2}$ Carmen Lucia Sahlit - \\ ${ }^{1}$ Universidade Federal do Mato Grosso/Faculdade de Arquitetura Engenharia e Tecnologia - FAET. \\ ${ }^{2}$ Universidade de Brasília/Faculdade de Tecnologia - FT.
}

Recebido em: Fevereiro 2015 Aceito em: Março de $2015 \quad$ Publicado em: Junho de 2015

\begin{abstract}
RESUMO: O comportamento de estruturas aporticadas com conexões semi-rígidas sujeitadas a cargas dinâmicas é estudado. É avaliado o efeito da flexibilidade das conexões nas freqüências naturais e nos correspondentes modos de vibração, bem como nos deslocamentos dinâmicos e tensões generalizadas. As conexões flexíveis são representadas por meio de molas rotacionais de comprimento nulo, com relação momento-rotação linear. As matrizes de massa e de rigidez são deduzidas como a soma das matrizes convencionais de elemento finitos e matrizes de correção que incorporam a flexibilidade das juntas. O problema de vibrações forçadas é resolvido então por meio do método de superposição modal ou, alternativamente, por integração numérica das equações de movimento. São apresentados resultados numéricos e para isto é mostrado que a consideração de conexões semi-rígidas altera o comportamento dinâmico da estrutura significativamente.
\end{abstract}

Palavras chave: Estrutura reticulada, conexão semi-rígida, resposta dinâmica, análise de vibrações.

ABSTRACT: The behaviour of framed structures with semi-rigid connections subjected to dynamic loads is studied. Attention is given to the effect of joint flexibility on the natural frequencies and corresponding mode shapes, as well as on the dynamic displacements and generalised stresses. The flexible connections are represented by zero-length rotational springs with linear moment-rotation relationship. The mass and stiffness matrices are developed as the sum of the conventional finite element matrices and correction matrices which incorporate the flexibility of the end joints. The problem of forced vibrations is then solved by means of the modal superposition method or, alternatively, by numerical integration of the motion equations. Numerical results are presented and it is shown that the consideration of semi-rigid connections alters significantly the dynamic behaviour of the structure.

Keywords: Framed structure, semi-rigid connection, dynamic response, vibration analysis.

\section{INTRODUÇÃO}

A conexão semi-rígida é aquela que possui uma capacidade de transmissão de momento intermediária entre a rígida e a flexível. Ela permite que, sob a ação de um carregamento, os elementos interligados apresentem uma rotação relativa $\mathrm{e}$ transmitam entre si parte do momento atuante. Pode-se modelar uma conexão viga-pilar de pórtico plano considerando os três graus de liberdade. No entanto, como a influência das deformações axial e cisalhante é , em geral, pequena em relação à deformação rotacional (Jones et al., 1983), o comportamento da conexão semirígida é descrito, no presente trabalho, pela relação entre o momento transmitido pela mesma e a rotação relativa entre os dois elementos interligados.

Apesar do comportamento da maioria das conexões não ser linear ao longo de toda a curva momento-rotação, a aproximação linear é, em geral, suficiente para a análise de pórticos submetidos a cargas de serviço (Lui \& Chen, 1987). Considera-se, portanto, a relação momento-rotação da conexão como sendo linear elástica.

O comportamento da conexão é um fator importante a ser considerado na análise de pórticos submetidos a cargas dinâmicas (Osman et al., 1993). Entretanto, a literatura sobre a análise dinâmica de estruturas semi-rígidas é limitada. Shi \& Atluri (1989) e Chan \& 
Ming Ho (1994) propuseram métodos para análise de vibrações livres de pórticos semi-rígidos, sem contudo explicitar os coeficientes das matrizes elementares envolvidas. Suarez et al. (1996) estudaram o comportamento de estruturas reticuladas com conexões flexíveis, submetidas a excitações sísmicas, descrevendo o comportamento da junta em função de sua flexibilidade e de sua excentricidade. Estes autores observaram, no entanto, que o efeito do comprimento ou excentricidade das conexões é muito pequeno, podendo ser desprezado. Soares Filho et al. (2002) estudou pórticos metálicos com juntas modeladas como elastoplásticas com encruamento. Soares Filho et al. (2003) realizou o controle de vibrações em estruturas semi-rígidas equipadas com Amortecedor de Massa Sintonizado

Figura 1-Elemento deformado com conexões semi-rigidas

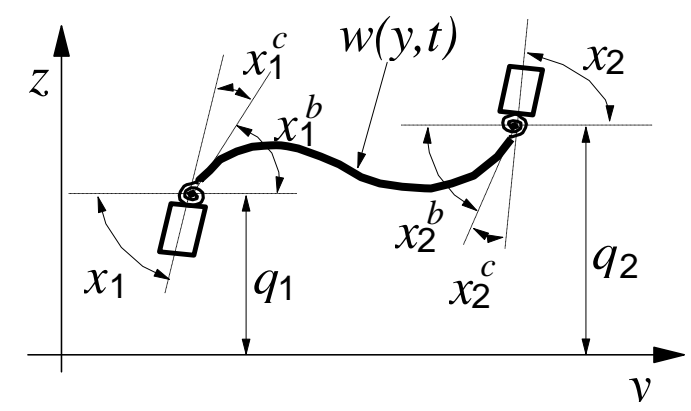

(AMS). Soares Filho et al. (2004) avaliou a ação de ventos em estruturas aporticadas semi-rígidas. Estes autores constataram mudanças importantes nas respostas estruturais.

O presente trabalho aborda a influência da modificação das matrizes de rigidez e de massa nos valores das frequiências e nos modos naturais de vibração de estruturas reticuladas semirígidas. Também realiza-se a análise de estruturas reticuladas com conexões semirígidas, submetidas a excitações dinâmicas. A estrutura é discretizada em elementos de barra de comprimento finito, identificados pelos seus nós extremos. Admite-se que as conexões tenham comprimento nulo e assume-se como válido o regime de deslocamentos e deformações infinitesimais. O material estrutural é considerado linear elástico e as relações constitutivas correspondentes são descritas na forma rigidez. Utilizando-se as funções de forma convencionais de um elemento de viga, obtêm-se as matrizes de massa e de rigidez, modificadas pela inclusão da flexibilidade das conexões semi-rígidas. Consideram-se os modelos de massa consistente e de massa concentrada para os elementos discretizados na análise estrutural. As matrizes globais para uma estrutura reticulada com juntas semirígidas são obtidas através da combinação das matrizes de todos os elementos. O problema de autovalores, associado a vibrações livres, é resolvido utilizando-se o método de Jacobi. O problema de vibrações forçadas é estudado através do método da superposição modal ou, alternativamente, pela integração numérica das equações de movimento. Apresentamse exemplos numéricos cujos resultados são comparados com os disponíveis na literatura, mostrando a validade das formulações desenvolvidas.

\section{MATRIZ DE RIGIDEZ MODIFICADA}

Considera-se o elemento estrutural, de comprimento $L$, com conexões semirígidas, indicado na Figura 1, onde $x_{i}^{b}$ é a deformação da barra, $x_{i}^{c}$ é a deformação da conexão e $x_{i}$ é a deformação total (rotação relativa entre os elementos ligados pela conexão), sendo $i$ a extremidade do elemento $(i=1,2)$. Observa-se na Figura 1 que

$$
x_{i}=x_{i}^{b}+x_{i}^{c}
$$

onde a deformação $x_{i}^{c}$, causada pela flexibilidade da conexão, depende da rigidez $k_{i}$ da mesma e do momento fletor atuante $X_{i}$, sendo igual a 


$$
x_{i}^{c}=\frac{X_{i}}{k_{i}}
$$

Para a análise dinâmica de um elemento de viga, pode-se definir o campo dos deslocamentos $w(y, t)$ em termos de quatro funções de forma $\Psi_{i}(y)$ e dos deslocamentos nodais $q_{1}(t), x_{1}^{b}(t), q_{2}(t) \mathrm{e}$ $x_{2}^{b}(t)$, no instante $t$, como:

$$
w(y, t)=\boldsymbol{\psi}(y) \boldsymbol{q}(t)=\left[\begin{array}{llll}
\Psi_{1}(y) & \Psi_{2}(y) & \Psi_{3}(y) & \Psi_{4}(y)
\end{array}\right]\left[\begin{array}{c}
q_{1}(t) \\
x_{1}^{b}(t) \\
q_{2}(t) \\
x_{2}^{b}(t)
\end{array}\right]
$$

onde

$$
\begin{array}{ll}
\Psi_{1}(y)=\frac{2 y^{3}}{L^{3}}-\frac{3 y^{2}}{L^{2}}+1 & , \quad \Psi_{2}(y)=\frac{y^{3}}{L^{2}}-\frac{2 y^{2}}{L}+y \\
\Psi_{3}(y)=-\frac{2 y^{3}}{L^{3}}+\frac{3 y^{2}}{L^{2}} \quad, & \Psi_{4}(y)=\frac{y^{3}}{L^{2}}-\frac{y^{2}}{L}
\end{array}
$$

Levando-se em conta a rotação adicional devida à conexão, equação (2), pode-se escrever o campo de deslocamentos (3)

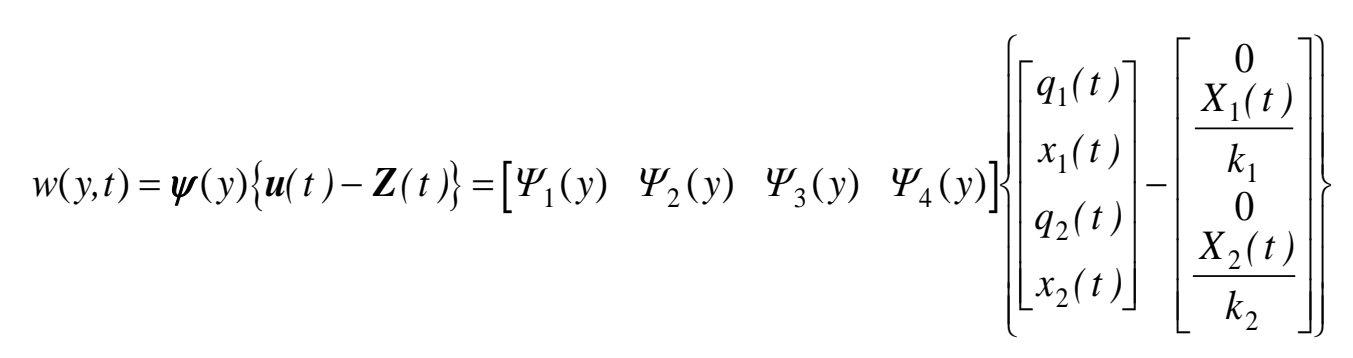

Considerando as funções de forma (4), e substituindo-se (5) em:

$$
X_{1}(t)=-\left.E I w^{\prime \prime}(y, t)\right|_{y=0} \quad, \quad X_{2}(t)=\left.E I w^{\prime \prime}(y, t)\right|_{y=L}
$$

chega-se a:

$$
\left[\begin{array}{cc}
1+4 W_{1} & 2 W_{2} \\
2 W_{1} & 1+4 W_{2}
\end{array}\right]\left[\begin{array}{l}
X_{1}(t) \\
X_{2}(t)
\end{array}\right]=\frac{E I}{L^{2}}\left[\begin{array}{cccc}
6 & 4 L & -6 & 2 L \\
6 & 2 L & -6 & 4 L
\end{array}\right]\left[\begin{array}{c}
q_{1}(t) \\
x_{1}(t) \\
q_{2}(t) \\
x_{2}(t)
\end{array}\right]
$$

onde $R_{i}=L k_{i} / E I=1 / W_{i}$ representa o índice de rigidez da conexão na extremidade $i(i=1,2)$.

A partir de (7), e fazendo-se $\Delta=\left[\left(1+4 W_{1}\right)\left(1+4 W_{2}\right)-4 W_{1} W_{2}\right]$, obtém-se:

$$
\left[\begin{array}{l}
X_{1}(t) \\
X_{2}(t)
\end{array}\right]=\frac{E I}{L^{2} \Delta}\left[\begin{array}{cccc}
6\left(1+2 W_{2}\right) & 4 L\left(1+3 W_{2}\right) & -6\left(1+2 W_{2}\right) & 2 L \\
6\left(1+2 W_{1}\right) & 2 L & -6\left(1+2 W_{1}\right) & 4 L\left(1+3 W_{1}\right)
\end{array}\right]\left[\begin{array}{l}
q_{1}(t) \\
x_{1}(t) \\
q_{2}(t) \\
x_{2}(t)
\end{array}\right]
$$


Levando-se (8) na expressão (5) do campo de deslocamentos $w(y, t)$ e fazendo-se:

$$
\boldsymbol{B}_{1}^{T}=\left[\begin{array}{llll}
-\frac{6}{L}\left(W_{1}+2 W_{1} W_{2}\right) & -4\left(W_{1}+3 W_{1} W_{2}\right) & \frac{6}{L}\left(W_{1}+2 W_{1} W_{2}\right) & -2 W_{1}
\end{array}\right]
$$

$\mathrm{e}$

$$
\boldsymbol{B}_{2}{ }^{T}=\left[\begin{array}{llll}
-\frac{6}{L}\left(W_{2}+2 W_{1} W_{2}\right) & -2 W_{2} & \frac{6}{L}\left(W_{2}+2 W_{1} W_{2}\right) & -4\left(W_{2}+3 W_{1} W_{2}\right)
\end{array}\right]
$$

obtém-se:

$$
\boldsymbol{Z}(t)=\frac{-1}{\Delta} \boldsymbol{B} \boldsymbol{u}(t)=\frac{-1}{\Delta}\left[\begin{array}{c}
\mathbf{0}^{T} \\
\boldsymbol{B}_{1}{ }^{T} \\
\mathbf{0}^{T} \\
\boldsymbol{B}_{2}{ }^{T}
\end{array}\right]\left[\begin{array}{c}
q_{1}(t) \\
x_{1}(t) \\
q_{2}(t) \\
x_{2}(t)
\end{array}\right]
$$

Pode-se então exprimir $w(y, t)$ em termos dos deslocamentos nodais $q_{1}(t)$ e $q_{2}(t)$ e

das rotações de extremidades $x_{1}(t)$ e $x_{2}(t)$ como:

$$
w(y, t)=\boldsymbol{\psi}(y)\left\{\boldsymbol{I}+\frac{1}{\Delta} \boldsymbol{B}\right\} \boldsymbol{u}(t)
$$

onde a matriz $\boldsymbol{B}$ reflete o efeito da flexibilidade das conexões semi-rígidas.

A expressão da energia potencial total $W_{T}$ para um elemento de viga, com rigidez à flexão $E I$, é composta de duas parcelas, $W_{v}$ e $W_{c}$, a primeira devida à deformação

elástica da viga, a segunda devida à flexibilidade rotacional da conexão, sendo as mesmas iguais a:

$$
W_{v}=\frac{1}{2} \int_{0}^{L} E I\left[w^{\prime \prime}(y, t)\right]^{2} d y \quad, \quad W_{c}=\frac{1}{2} k_{1}\left[x_{1}^{c}\right]^{2}+\frac{1}{2} k_{2}\left[x_{2}^{c}\right]^{2}
$$

Substituindo-se a segunda derivada de (12) em relação a $y$ na equação (13a), chega-se a:

$$
W_{v}=\frac{1}{2}[\boldsymbol{u}(t)]^{T}\left[\boldsymbol{I}+\frac{1}{\Delta} \boldsymbol{B}^{T}\right]\left\{\int_{0}^{L} E I\left[\boldsymbol{\psi}^{\prime \prime}(y)\right]^{T}\left[\boldsymbol{\psi}^{\prime \prime}(y)\right] d y\right\}\left[\boldsymbol{I}+\frac{1}{\Delta} \boldsymbol{B}\right] \boldsymbol{u}(t)
$$

Sabendo-se que a integral em (14) representa a matriz de rigidez convencional $\boldsymbol{K}_{0}$ (por exemplo, Clough \&

$$
W_{v}=\frac{1}{2}[\boldsymbol{u}(t)]^{T}\left\{\boldsymbol{K}_{0}+\boldsymbol{K}_{1}\right\} \boldsymbol{u}(t)
$$

onde

$$
\boldsymbol{K}_{1}=\boldsymbol{K}_{0} \frac{1}{\Delta} \boldsymbol{B}+\frac{1}{\Delta} \boldsymbol{B}^{T} \boldsymbol{K}_{0}+\frac{1}{\Delta} \boldsymbol{B}^{T} \boldsymbol{K}_{0} \frac{1}{\Delta} \boldsymbol{B}
$$

Penzien, 1993), pode-se reescrever a expressão da energia potencial devida à deformação elástica da viga como:

Levando-se em conta (2), (8) e (11) obtém-se:

$$
x_{i}^{c}=\frac{-1}{\Delta} \boldsymbol{B}^{T} \boldsymbol{u}(t)
$$

Substituindo-se (17) em (13b), pode-se reescrever a expressão da energia potencial devida à flexibilidade das conexões como: 


$$
W_{c}=\frac{1}{2}[\boldsymbol{u}(t)]^{T}\left[k_{1} \boldsymbol{B}_{1} \boldsymbol{B}_{1}^{T}+k_{2} \boldsymbol{B}_{2} \boldsymbol{B}_{2}{ }^{T}\right] \frac{\boldsymbol{u}(t)}{\Delta^{2}}=\frac{1}{2}[\boldsymbol{u}(t)]^{T}\left[\boldsymbol{K}_{2}\right] \frac{\boldsymbol{u}(t)}{\Delta^{2}}
$$

A expressão da energia potencial total é, portanto,

$$
W_{T}=W_{v}+W_{c}=\frac{1}{2}[\boldsymbol{u}(t)]^{T}\left\{\boldsymbol{K}_{0}+\boldsymbol{K}_{1}+\boldsymbol{K}_{2}\right\} \boldsymbol{u}(t)
$$

onde $\boldsymbol{K}_{1}$ e $\boldsymbol{K}_{2}$ representam as matrizes de correção da matriz de rigidez convencional $\boldsymbol{K}_{0}$ para se levar em consideração a presença de conexões semi-rígidas. A matriz de rigidez $\overline{\boldsymbol{K}}$, modificada pela inclusão de juntas semi-rígidas, é dada

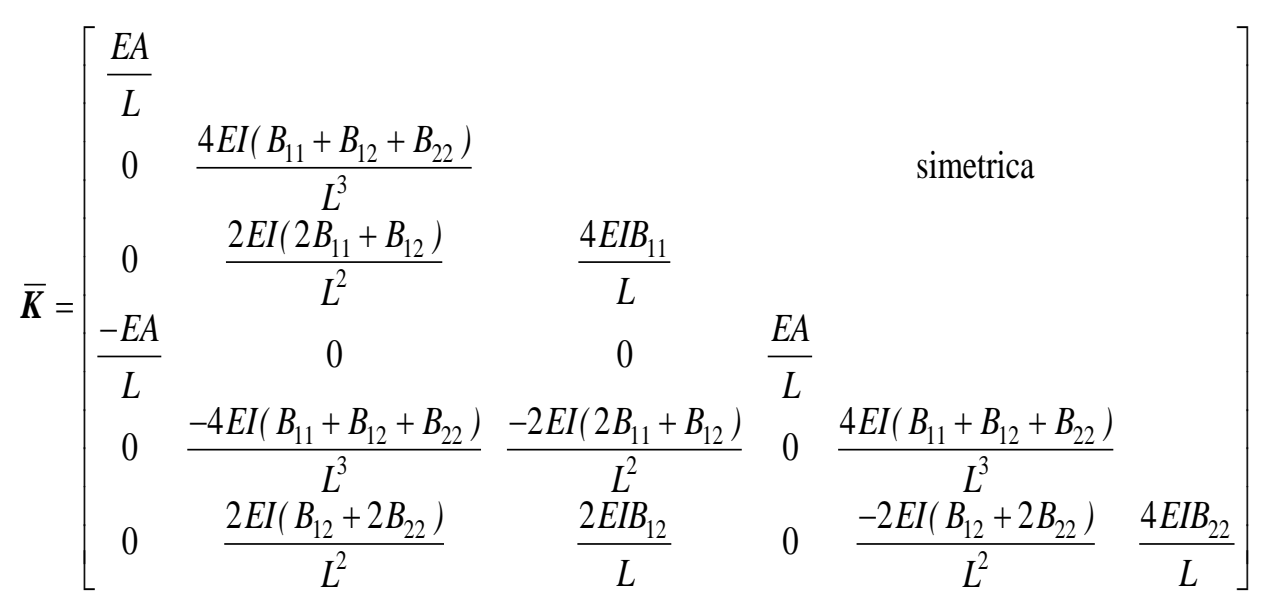

onde

$$
B_{11}=\frac{3 P_{1}}{4-P_{1} P_{2}} \quad, \quad B_{12}=\frac{3 P_{1} P_{2}}{4-P_{1} P_{2}} \quad, \quad B_{22}=\frac{3 P_{2}}{4-P_{1} P_{2}}
$$

conexões forem rígidas, então $P_{1}=P_{2}=1.0$ e a matriz $\overline{\boldsymbol{K}}$ se reduz à matriz de rigidez convencional.

com juntas semi-rígidas, utiliza-se a expressão da energia cinética $T$ para um elemento de viga com massa por unidade de comprimento $\bar{m}$, a qual é:

$$
T=\frac{1}{2} \int_{0}^{L} \bar{m}(y)[\dot{w}(y, t)]^{2} d y
$$

Fazendo-se a primeira derivada em relação ao tempo da expressão (12) do campo de

portanto pela soma das três matrizes $\boldsymbol{K}_{0}, \boldsymbol{K}_{1}$ e $\boldsymbol{K}_{2}$. Como a presença das conexões semicorrespondentes aos efeitos axiais, a matriz de rigidez de um elemento desconexo com juntas semi-rígidas é, finalmente,

$$
T=\frac{1}{2}[\dot{\boldsymbol{u}}(t)]^{T}\left[\boldsymbol{I}+\frac{1}{\Delta} \boldsymbol{B}^{T}\right]\left\{\int_{0}^{L} \bar{m}(y)[\boldsymbol{\psi}(y)]^{T} \boldsymbol{\psi}(y) d y\right\}\left[\boldsymbol{I}+\frac{1}{\Delta} \boldsymbol{B}\right] \dot{\boldsymbol{u}}(t)
$$

A integral em (23) denota a matriz de massa consistente convencional $\boldsymbol{M}_{0}$ (por exemplo, Clough \& Penzien, 1993), podendo-se reescrever (23) como: 


$$
T=\frac{1}{2}[\dot{\boldsymbol{u}}(t)]^{T}\left\{\boldsymbol{M}_{0}+\boldsymbol{M}_{0} \frac{1}{\Delta} \boldsymbol{B}+\frac{1}{\Delta} \boldsymbol{B}^{T} \boldsymbol{M}_{0}+\frac{1}{\Delta} \boldsymbol{B}^{T} \boldsymbol{M}_{0} \frac{1}{\Delta} \boldsymbol{B}\right\} \dot{\boldsymbol{u}}(t)
$$

A energia cinética é então expressa como:

$$
T=\frac{1}{2}[\dot{\boldsymbol{u}}(t)]^{T}\left\{\boldsymbol{M}_{0}+\boldsymbol{M}_{1}\right\} \dot{\boldsymbol{u}}(t)
$$

Onde:

$$
\boldsymbol{M}_{1}=\boldsymbol{M}_{0} \frac{1}{\Delta} \boldsymbol{B}+\frac{1}{\Delta} \boldsymbol{B}^{T} \boldsymbol{M}_{0}+\frac{1}{\Delta} \boldsymbol{B}^{T} \boldsymbol{M}_{0} \frac{1}{\Delta} \boldsymbol{B}
$$

representa a matriz de correção da matriz de massa consistente convencional para se levar em consideração a presença de conexões semi-rígidas. A matriz de massa

Da mesma forma que na matriz de rigidez, a presença das juntas semi-rígidas não afeta os coeficientes de rigidez

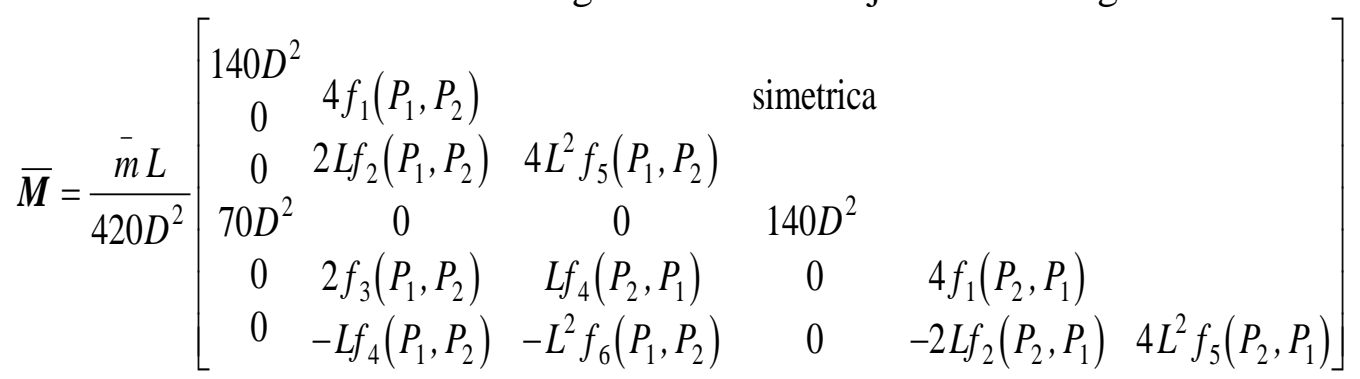

consistente $\overline{\boldsymbol{M}}$ modificada pela inclusão de juntas semi-rígidas, é dada portanto pela soma das duas matrizes $\boldsymbol{M}_{0}$ e $\boldsymbol{M}_{1}$.

relacionados aos efeitos axiais. Assim sendo, a matriz de massa de um elemento com juntas semi-rígidas é:

Onde:

$D=4-P_{1} P_{2}$

$f_{1}\left(P_{1}, P_{2}\right)=\left(560+224 P_{1}+32 P_{1}^{2}-196 P_{2}-328 P_{1} P_{2}-55 P_{1}^{2} P_{2}+32 P_{2}^{2}+50 P_{1} P_{2}^{2}+32 P_{1}^{2} P_{2}^{2}\right)$

$f_{2}\left(P_{1}, P_{2}\right)=\left(224 P_{1}+64 P_{1}^{2}-160 P_{1} P_{2}-86 P_{1}^{2} P_{2}+32 P_{1} P_{2}^{2}+25 P_{1}^{2} P_{2}^{2}\right)$

$f_{3}\left(P_{1}, P_{2}\right)=\left(560-28 P_{1}-64 P_{1}^{2}-28 P_{2}-184 P_{1} P_{2}+5 P_{1}^{2} P_{2}-64 P_{2}^{2}+5 P_{1} P_{2}^{2}+41 P_{1}^{2} P_{2}^{2}\right)$

$f_{4}\left(P_{1}, P_{2}\right)=\left(392 P_{2}-100 P_{1} P_{2}-64 P_{1}^{2} P_{2}-128 P_{2}^{2}-38 P_{1} P_{2}^{2}+55 P_{1}^{2} P_{2}^{2}\right)$

$f_{5}\left(P_{1}, P_{2}\right)=\left(32 P_{1}^{2}-31 P_{1}^{2} P_{2}+8 P_{1}^{2} P_{2}^{2}\right)$

$f_{6}\left(P_{1}, P_{2}\right)=\left(124 P_{1} P_{2}-64 P_{1}^{2} P_{2}-64 P_{1} P_{2}^{2}+31 P_{1}^{2} P_{2}^{2}\right)$

Ressalta-se ainda que quando $P_{i}$ assume o valor 1.0 (engaste perfeito) ou 0.0 (rótula

EQUAÇÃO DE MOVIMENTO PARA ANÁLISE DE VIBRAÇÕES FORÇADAS

A equação de movimento para a análise de vibrações forçadas de uma estrutura com conexões semi-rígidas, expressa matricialmente, é dada por

$$
\overline{\boldsymbol{M}}_{g} \ddot{\boldsymbol{u}}(t)+\overline{\boldsymbol{C}}_{g} \dot{\boldsymbol{u}}(t)+\overline{\boldsymbol{K}}_{g} \boldsymbol{u}(t)=\boldsymbol{\lambda}_{g}(t)
$$

perfeita), a matriz $\overline{\boldsymbol{M}}$ se reduz à matriz de massa consistente convencional $\boldsymbol{M}_{0}$. 
é o vetor, de ordem ( $n \times 1)$, de cargas nodais aplicadas. Convém salientar que, no caso de cargas distribuídas ao longo dos elementos, o vetor $\lambda_{g}(t)$ deve ser também modificado para levar em conta a flexibilidade das juntas, como descrito, por exemplo, em Wang (1989).

O sistema (29) é resolvido, no presente trabalho, pelo método da superposição modal ou, alternativamente pela integração numérica utilizando-se o esquema de Newmark.

Quando não houver forças aplicadas na estrutura, $\lambda_{g}(t)=\mathbf{0}$, a solução de (29) recai em um problema característico ou de autovalor, cuja resolução fornece os valores das freqüências naturais $\omega_{j}$ e correspondentes modos naturais de vibração $\phi_{j}(j=1, \ldots, n)$. No presente trabalho utiliza-se o método de Jacobi para o cálculo dos autovalores e autovetores associados (Clough \& Penzien, 1993).

\section{EXEMPLOS NUMÉRICOS}

Nesta seção apresentam-se dois exemplos de análise de vibrações de pórticos planos com conexões semirígidas, considerando-se que a junta se comporte elasticamente. Tem-se o intuito de validar a formulação desenvolvida e a implementação computacional realizada.

\section{Exemplo 1:}

Considera-se uma viga simples, anteriormente analisada por Chan (1994), apresentando as seguintes propriedades: $A=27 \times 10^{-3} \mathrm{~m}^{2}, E=210 \times 10^{6}$ $\mathrm{Kn} / \mathrm{m}^{2}, I=50 \times 10^{-6} \mathrm{~m}^{4}, \rho=78.255 \mathrm{Kn} / \mathrm{m}^{3} \mathrm{e}$ $L=1.0 \mathrm{~m}$. Objetiva-se determinar o valor da frequiência fundamental de vibração, levando-se em conta quatro situações de fixação de extremidade, quais sejam, engaste-engaste (E-E), engaste-rótula (E$\mathrm{R}$ ), rótula-rótula (R-R) e semi-rígidas. $\mathrm{Na}$ situação em que se considera extremidades com juntas semi-rígidas o fator de fixação $P_{i}$ utilizado é igual a 0.25 .

Para uma viga simples, sabe-se que o valor analítico exato da frequiência fundamental é dado por $\omega=\bar{C} \sqrt{E I / \bar{m} L^{4}}$. Nesta expressão, o parâmetro $\bar{C}$, que é função das condições de fixação das extremidades da viga, tem o valor $\bar{C}$ $=22.3733$ no caso (E-E), $\bar{C}=15.4118$ no caso (E-R) e $\bar{C}=\pi^{2}$ no caso (R-R). Para o caso de junta semi-rígida, contudo, não se tem o valor analítico da frequiência fundamental.

As Tabelas 1 e 2 apresentam os valores encontrados para a freqüência fundamental nos casos de massa consistente e de massa concentrada, respectivamente. Nas mesmas tabelas, indica-se o erro percentual $(\boldsymbol{\varepsilon} \%)$ relativo ao valor analítico exato, considerando-se a viga devidamente discretizada $\mathrm{e}$ as condições de apoio (E-E), (E-R) e (R-R). Como não se conhece, no caso de apoio semi-rígido, o valor exato da freqüência fundamental, adota-se como referência o valor encontrado para a discretização mais refinada.

Conforme indicado na literatura (por exemplo, Araújo \& Venâncio Filho, 1971), as freqüências naturais obtidas com o uso de matriz de massa consistente constituem limites superiores das freqüências naturais exatas, enquanto que as obtidas com massa concentrada constituem limites inferiores das mesmas. Esta constatação pode ser corroborada pela análise dos resultados apresentados nas referidas tabelas. Verifica-se ainda que a discretização em oito elementos fornece excelentes resultados para o valor da freqüência fundamental nas várias situações de fixação das extremidades da viga, em ambos os modelos de massa adotados. 
Soares M. F., Sahlit C. L., Comportamento Dinâmico de Estruturas Reticuladas com Juntas Semi-Rigidas. E\&S - Engineering and Science, (2015), 3:1.

Tabela 1: Valores da freqüência fundamental - matriz de massa consistente modificada

\begin{tabular}{|c|c|c|c|c|c|c|c|}
\hline \multirow[b]{2}{*}{ situação } & \multicolumn{2}{|c|}{2 elementos } & \multicolumn{2}{|c|}{4 elementos } & \multicolumn{2}{|c|}{8 elementos } & \multirow{2}{*}{$\begin{array}{l}\text { Valor } \\
\text { Exato }\end{array}$} \\
\hline & $\omega(\mathrm{rd} / \mathrm{s})$ & $\varepsilon \%$ & $\omega(\mathrm{rd} / \mathrm{s})$ & $\varepsilon \%$ & $\begin{array}{c}\omega \\
(\mathrm{rd} / \mathrm{s})\end{array}$ & $\varepsilon \%$ & \\
\hline E-E & 1602.760 & 1.6206 & 1579.290 & 0.1325 & $\begin{array}{r}1577.38 \\
0\end{array}$ & 0.0114 & 1577.200 \\
\hline E-R & 1103.310 & 1.5517 & 1087.660 & 0.1113 & $\begin{array}{r}1086.94 \\
0\end{array}$ & 0.0450 & 1086.451 \\
\hline $\begin{array}{l}\text { semi- } \\
\text { rígida }\end{array}$ & 821.867 & 0.9414 & 814.633 & 0.0529 & 814.202 & referência & --- \\
\hline $\mathbf{R}-\mathbf{R}$ & 700.788 & 0.7234 & 695.960 & 0.0295 & 695.730 & 0.0036 & 695.755 \\
\hline
\end{tabular}

Tabela 2: Valores da freqüência fundamental - matriz de massa concentrada

\begin{tabular}{|c|c|c|c|c|c|c|c|}
\hline \multirow[b]{2}{*}{ situação } & \multicolumn{2}{|c|}{4 elementos } & \multicolumn{2}{|c|}{8 elementos } & \multicolumn{2}{|c|}{16 elementos } & \multirow{2}{*}{$\begin{array}{c}\text { Valor } \\
\text { Analítico }\end{array}$} \\
\hline & $\omega$ & $\varepsilon \%$ & $\omega$ & $\varepsilon \%$ & $\omega$ & $\varepsilon \%$ & \\
\hline E-E & 1526.950 & 3.1860 & 1564.580 & 0.8002 & 1573.930 & 0.2073 & 1577.200 \\
\hline E-R & 1065.650 & 1.9146 & 1080.210 & 0.5744 & 1084.970 & 0.1363 & 1086.451 \\
\hline semi-rígida & 802.262 & 1.4734 & 810.209 & 0.4974 & 813.164 & referência & --- \\
\hline $\mathrm{R}-\mathrm{R}$ & 686.761 & 1.2927 & 692.401 & 0.4821 & 694.832 & 0.1327 & 695.755 \\
\hline
\end{tabular}

Em seguida, verifica-se a variação do valor da freqüência fundamental quando se varia o valor de $R_{i}$, índice de rigidez da conexão, do valor zero (caso de rótula perfeita) até o valor infinito (caso de engaste perfeito). Consideram-se dois casos de fixação de extremidade: no primeiro, ambas as extremidades são semirígidas (com $R_{i}$ variável); no segundo, uma extremidade é semi-rígida e a outra permanece como apoio simples $\left(R_{i}=0.0\right)$. Adota-se a discretização da viga em oito elementos, uma vez que se verificou ser a mesma suficiente para a obtenção de valores excelentes para a freqüência fundamental.

A Figura 2 mostra a variação do parâmetro $\bar{C}=\omega / \sqrt{E I / \bar{m} L^{4}}$ quando a rigidez da conexão varia do valor zero ao infinito, para cada um dos dois casos em estudo. Pode-se verificar claramente que o valor de $\bar{C}$ tende a se aproximar do valor analítico exato $(\bar{C}=22.3733$ no caso E-E e $\bar{C}=15.4118$ no caso E-R) quando a rigidez da conexão tende ao infinito. Vale ressaltar que as curvas indicadas na Figura 2 coincidem com as fornecidas por Chan (1994). 
Figura 2: Freqüência fundamental versus rigidez da conexão

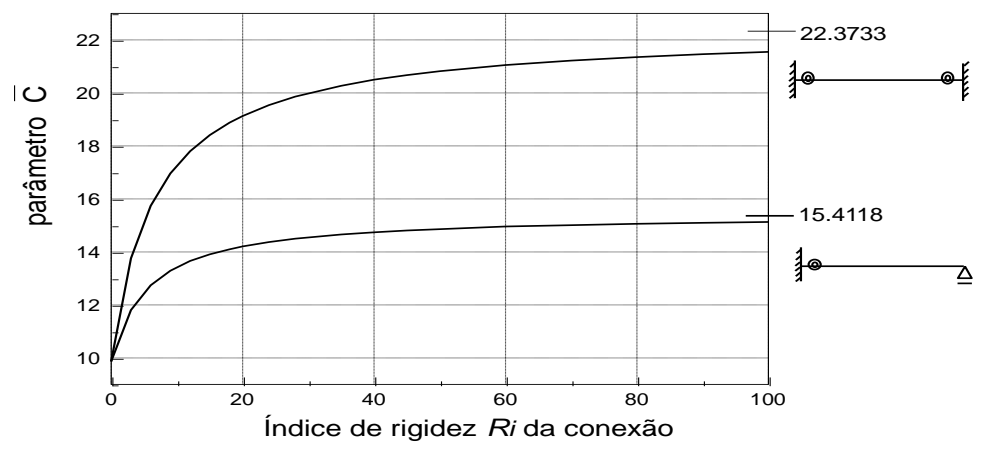

Figura 3-Pórtico metálico de 10 pavimentos.

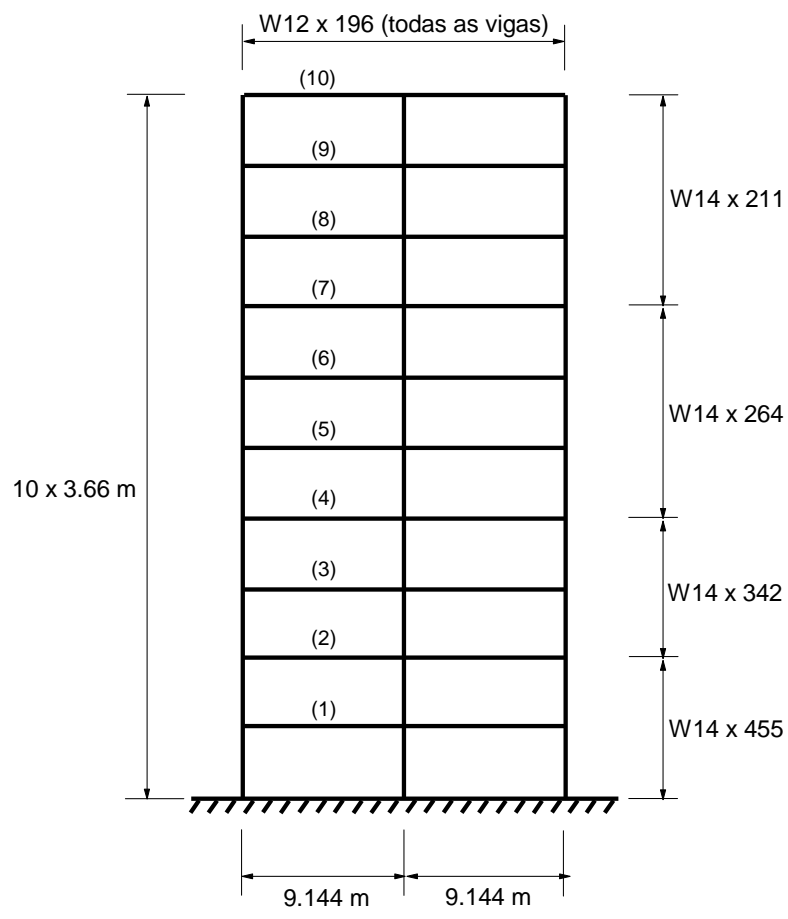

Exemplo 2 - Considera-se o pórtico metálico de dez pavimentos da Figura 3, estudado anteriormente por Suarez et al. (1996), com conexões semi-rígidas somente nas ligações das vigas com os pilares. Os perfis metálicos utilizados estão indicados na referida figura, bem como as dimensões de cada peça. O módulo de elasticidade e a densidade do aço utilizados são, respectivamente, $E=$ $210.00 \times 10^{6} \mathrm{KN} / \mathrm{m}^{2}$ e $\rho=78.255 \mathrm{KN} / \mathrm{m}^{3}$. Objetiva-se estudar a influência das conexões semi-rígidas no que diz respeito aos valores das freqüências naturais de vibração e formas modais correspondentes. A Figura 4(a) indica a variação do valor normalizado das cinco primeiras frequiências naturais de vibração ao se modificar a rigidez das conexões. Conforme se pode observar, a flexibilidade das conexões altera significantemente os valores das freqüências naturais, sendo esta influência mais acentuada nas frequiências mais baixas.

A Figura 4(b) mostra os deslocamentos modais dos nós da coluna esquerda da estrutura, permitindo que se verifique a variação sofrida pelo primeiro modo natural de vibração ao se considerar a flexibilidade das conexões com fatores de fixação $P_{i}=0.1, P_{i}=0.5$ e $P_{i}=1.0$. Para o primeiro modo de vibração em estudo, quanto mais flexível for a conexão, tanto maiores serão os deslocamentos modais nos pavimentos mais altos, ocorrendo o contrário nos andares mais baixos. 
Soares M. F., Sahlit C. L., Comportamento Dinâmico de Estruturas Reticuladas com Juntas Semi-Rigidas. E\&S - Engineering and Science, (2015), 3:1.

Figura 4: Freqüências e Primeiro Modo de Vibração

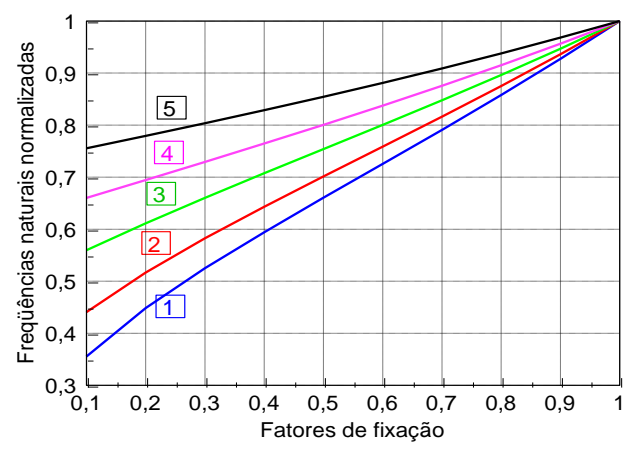

(a) Efeito da flexibilidade das juntas nas freqüências

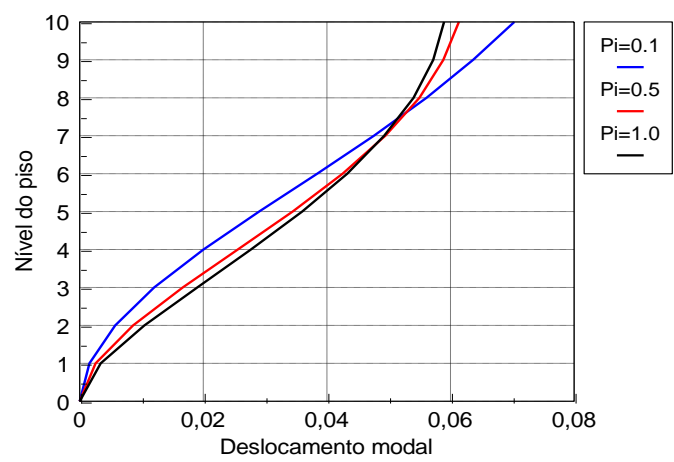

(b) Variação do primeiro modo de vibração

deslocamentos e esforços internos.

Figura 5-Pórtico plano para análise de vibrações forçadas.

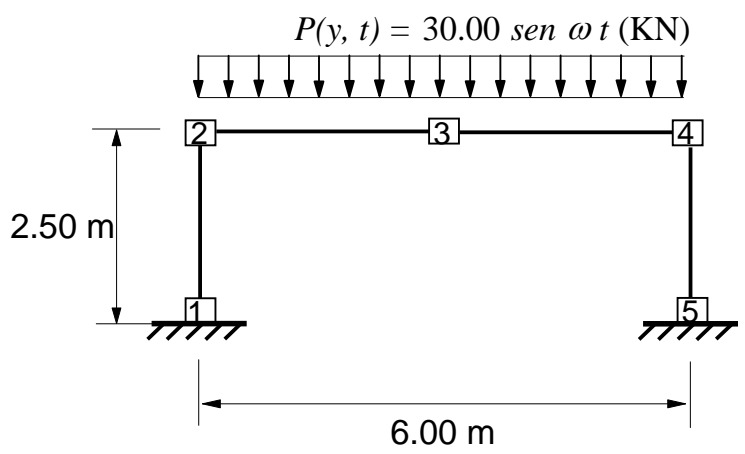

Convém salientar que os valores das frequiências e a primeira forma modal de vibração encontrados no presente trabalho, para os diversos fatores de fixação considerados, coincidem com os apresentados em Suarez et al. (1996).

Exemplo 3 - Considera-se o pórtico plano, indicado na Figura 5, com as seguintes propriedades: $A=240 \times 10^{-3}$ $\mathrm{m}^{2}, E=30 \times 10^{3} \mathrm{KN} / \mathrm{m}^{2}, I=0.0128 \mathrm{~m}^{4}$, $\bar{m}_{\text {viga }}=3.0581 \mathrm{KN} \mathrm{s} / \mathrm{m}^{2}$ e $\bar{m}_{\text {pilar }}=$ $0.5872 \mathrm{KN} \mathrm{s}^{2} / \mathrm{m}^{2}$. A estrutura apresenta uma taxa de amortecimento $\xi=0.05$ e está submetida a uma carga dinâmica vertical uniformemente distribuída, de valor $P(y, t)=30.0$ sen $100.0 t(\mathrm{KN})$. As conexões semirígidas são consideradas somente nas ligações das vigas com os pilares. Objetiva-se fazer a análise dinâmica da estrutura, estudando-se a influência da flexibilidade das conexões nos

As evoluções do deslocamento vertical do nó 3, obtidas pelo método da superposição modal e pelo esquema de Newmark, são indicadas na Figura $6 . \quad$ São considerados quatro fatores de fixação de extremidade, ocorrendo deslocamentos máximos iguais a $0.0827 \mathrm{~m}$ para $P_{i}=0.0,0.0671 \mathrm{~m}$ para $P_{i}=0.4,0.0593 \mathrm{~m}$ para $P_{i}=0.7 \mathrm{e}$ $0.0503 \mathrm{~m}$ para $P_{i}=1.0$. Observa-se que os deslocamentos e os períodos de vibração são tanto maiores quanto mais flexíveis forem as conexões, havendo consequientemente uma defasagem entre os instantes de deslocamentos máximos. Verifica-se ainda que as evoluções do deslocamento, no exemplo em questão, são igualmente amortecidas, qualquer que seja o fator de fixação considerado. 
Figura 6 - Evoluções do Deslocamento do Nó 3.

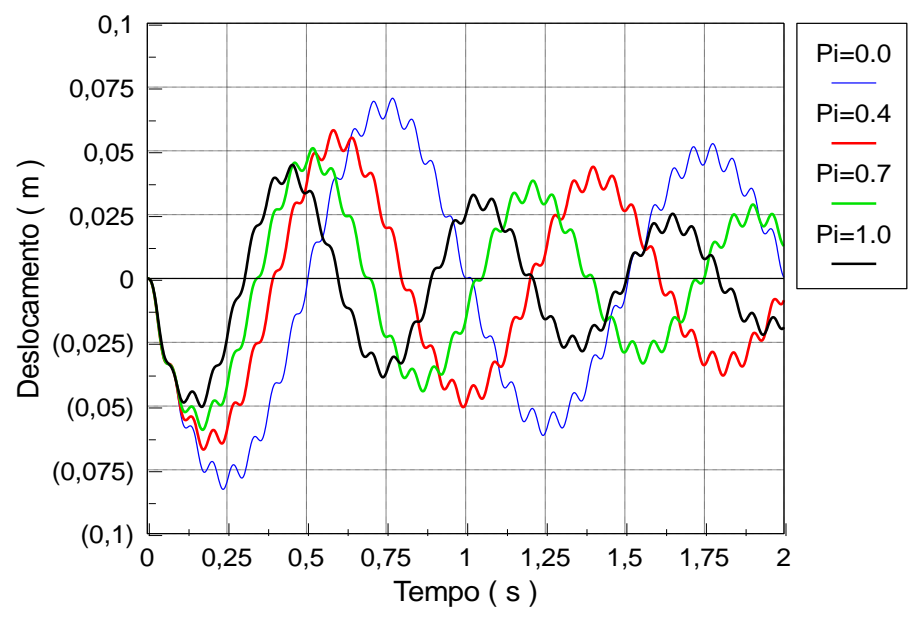

Na Tabela 3, pode-se observar o efeito da flexibilidade das conexões nos valores dos momentos fletores máximos em três seções distintas da estrutura. Verifica-se que os momentos fletores máximos, os quais atuam nos pontos críticos da viga, ou seja, no meio do vão para conexões rotuladas e nas extremidades no caso de engaste, tornam-se mais equilibrados quando são adotadas conexões semirígidas. Esta constatação pode resultar no dimensionamento de uma viga mais leve. Entretanto, para o dimensionamento ótimo do pórtico deve-se levar em conta que os momentos nos pilares também sofrem alteração.

Tabela 3: Momentos Fletores Máximos (kN x m)

\begin{tabular}{crrrr}
\hline \multicolumn{5}{c}{ fator de fixação da conexão } \\
\hline nó & \multicolumn{1}{c}{$P_{i}=0.0$} & $P_{i}=0.4$ & $P_{i}=0.7$ & $P_{i}=1.0$ \\
1 & 0.0000 & 1.0075 & 3.4055 & 5.0891 \\
2 & 0.0000 & 3.5175 & 6.1030 & 8.5784 \\
3 & 11.8674 & 10.4819 & 9.4273 & 9.8101 \\
\hline
\end{tabular}

\section{CONCLUSÕES}

No presente trabalho verificou-se que a alteração da frequiência natural de vibração, e conseqüente modificação das formas modais, pode ser significante quando se considera a semi-rigidez das conexões na análise estrutural. Observouse ainda que esta alteração é mais acentuada no que diz respeito às frequiências de vibração mais baixas. No caso das vibrações forçadas, os deslocamentos máximos e as distribuições de esforços sofrem mudanças importantes. Estes resultados indicam que a não consideração da flexibilidade intermediária das juntas entre os casos extremos, rótula e engaste, pode introduzir erros inaceitáveis na análise estrutural. Finalmente, concluise que, para se incluir o efeito das conexões semi-rígidas na análise de 
vibrações, são necessárias, de um modo geral, modificações nas matrizes de massa

\section{REFERÊNCIAS}

CHAN, S. L. \& MING HO, G. W., NonlinearVibration Analysis of Steel Frames with Semi-Rigid Connections, Journal of Structural Engineering, vol.120, pp.1075-1087, 1994.

CLOUGH, R. W. \& PENZIEN, J., Dynamics of Structures, 2ed., McGrawHill, Inc, New York, 1993.

JONES, S. W., KIRBY, P. A. \& NETHERCOT, D. A., The Analysis of Frames with Semi-Rigid Connections A State-of-the-Art Report, Journal of Constructional Steel Research, vol.3(2), pp.2-13, 1983.

LUI, E. M. \& CHEN, W. F., Steel Frame Analysis with Flexible Joints, Journal of Constructional Steel Research, vol.8, pp.161-202, 1987.

OSMAN, A., GHOBARAH, A. \& KOROL, R. M., Seismic Performance of Moment Resisting Frames with Flexible Joints, Engineering Structures, vol.15, pp.117-134, 1993.

SHI, G. \& ATLURI, S. N., Static and Dynamic Analysis of Space Frames With Non-Linear Flexible Connections, International Journal for Numerical Methods in Enginnering, vol.28, pp.2635-2650, 1989.

SOARES FILHO, M., SAHLIT, C. L. \& BRITO, J. L. V., "Comportamento Dinâmico de Pórticos Metálicos com Juntas Modeladas como Elastoplásticas com Encruamento", XXX Jornadas SulAmericanas de Engenharia Estrutural, CD-ROM, Brasília - DF, Brasil, 2002.

SOARES FILHO, M., SAHLIT, C. L. \& AVILA, S. M., (2003). "Controle de Vibrações em Estruturas Semi-Rígidas Equipadas com Amortecedor de Massa Sintonizado (AMS)", XXIV Iberian Latin-American Congress on Computational Methods in Engineering - CILAMCE, CD-ROM, Ouro Preto, Brazil, 2003. e de rigidez.

SOARES FILHO, M., GUIMARÃES, M. J. R., SAHLIT, C. L. \& BRITO, J. L. V., "Wind Pressures in Framed Structures with Semi-rigid Connections", Journal of The Brazilian Society of Mechanical Sciences \& Engineering, vol.XXVI (2), pp.180189, 2004.

SUAREZ, L. E., SINGH, M. P. \& MATHEU, E. E., Seismic Response of Structural Frameworks with Flexible Connections, Computer \& Structures, vol.58, pp.27-41, 1996.

WANG, C. K., Intermediate Structural Analysis, 6ed., Mc Grawl-Hill, chapter 20, pp.721-741, 1989. 